\title{
Composição Corporal e Exigências Nutricionais de Macrominerais para Cordeiros Morada Nova ${ }^{1}$
}

\section{Severino Gonzaga Neto ${ }^{2}$, Américo Garcia da Silva Sobrinho ${ }^{3}$, Kleber Tomás de Resende ${ }^{3}$, Nívea Maria Brancacci Lopes Zeola ${ }^{4}$, Aderbal Marcos de Azevedo Silva ${ }^{5}$, Carlo Aldrovandi Torreão Marques ${ }^{4}$, Luiz Gustavo Rombola ${ }^{6}$}

RESUMO - Este trabalho foi desenvolvido para se determinar a composição corporal e as exigências nutricionais de cálcio (Ca), fósforo $(\mathrm{P})$, magnésio $(\mathrm{Mg})$, sódio (Na) e potássio de ovinos da raça Morada Nova. Foram utilizados 30 cordeiros com peso vivo (PV) médio inicial de $15 \mathrm{~kg}$ e 70 dias de idade. Seis cordeiros foram abatidos aos $15 \mathrm{~kg}$, para determinação da composição corporal inicial (animais-referência) pela metodologia do abate comparativo; seis foram abatidos aos $20 \mathrm{~kg}$ (abate intermediário) e os demais distribuídos em seis grupos de três animais (um para cada dieta), de acordo com as relações volumoso(V):concentrado(C): 1) 40V:60C; 2) 55V:45C; e 3) 70V:30C. Os animais, em cada grupo, foram abatidos quando o cordeiro que recebia a dieta com maior teor de concentrado atingiu $25 \mathrm{~kg}$ de $\mathrm{PV}$. A composição corporal variou de 14,33 a 12,42 g de Ca; 8,12 a 7,15 g de P; 0,47 a 0,46 g de Mg; 1,60 a 1,40 g de Na e de 2,30 a 2,23 g de K por kg de peso de corpo vazio. As exigências líquidas de ganho variaram de 13,54 a 11,74 mg de Ca; 7,96 a 7,02 mg de P; 0,57 a $0,55 \mathrm{mg}$ de $\mathrm{Mg} ; 1,54$ a 1,35 mg de $\mathrm{Na}$ e de 2,75 a 2,68 mg de K por g de ganho de PV. As exigências dietéticas diárias de macrominerais para cordeiros dos 15 aos $25 \mathrm{~kg}$ de PV, ganhando $100 \mathrm{~g} / \mathrm{dia}$, variaram de 2,76 a 3,12 g de Ca; 1,91 a 2,95 de g de P; 0,60 a 0,77 g de Mg; 0,60 a $0,86 \mathrm{~g}$ de $\mathrm{Na}$ e de 1,51 a 2,63 g de $\mathrm{K}$.

Palavras-chave: cálcio, exigências, fósforo, magnésio, potássio, sódio

\section{Body Composition and Nutritional Requirements of Macrominerals for Morada Nova Sheep}

\begin{abstract}
The objective of this trial was to determine the body composition and nutritional requirements of macrominerals for Morada Nova sheep. Thirty lambs averaging $15 \mathrm{~kg}$ of initial body weight (BW) and 70 days of age were used in this study. Six lambs (reference-animals) were slaughtered with $15 \mathrm{~kg}$ of BW to determine the initial body composition using the comparative slaughter methodology; six other lambs were slaughtered with $20 \mathrm{~kg}$ of BW (intermediary slaughter) and the remaining 18 lambs were distributed to six groups of three animals (one lamb for each treatment) and received diets with the following forage(F):concentrate(C) ratio: 1) 40F:60C;2) 55F:45C; and 3) 70F:30C. Animals from each group were slaughtered when the lamb that had been fed the diet with the highest concentrate level reached $25 \mathrm{~kg}$ of BW. Body composition varied from 14.33 to $12.42 \mathrm{~g}$ of Ca, 8.12 to $7.15 \mathrm{~g}$ of $\mathrm{P}, 0.47$ to $0.46 \mathrm{~g}$ of $\mathrm{Mg}, 1.60$ to $1.40 \mathrm{~g}$ of $\mathrm{Na}$, and 2.30 to $2.23 \mathrm{~g}$ of $\mathrm{K}$ per $\mathrm{kg}$ of empty body weight. Net gain requirements ranged from 13.54 to $11.74 \mathrm{mg}$ of Ca, 7.96 to $7.02 \mathrm{mg}$ of $\mathrm{P}, 0.57 \mathrm{mo} 0.55 \mathrm{mg}$ of $\mathrm{Mg}, 1.54$ to 1.35 $\mathrm{mg}$ of $\mathrm{Na}$, and 2.75 to $2.68 \mathrm{mg}$ of K per g of gained BW. Daily macromineral requirements for lambs from 15 to $25 \mathrm{~kg}$ of BW gaining $100 \mathrm{~g} / \mathrm{day}$ varied from 2.76 to $4.74 \mathrm{~g} \mathrm{Ca}, 1.91$ to $3.15 \mathrm{~g}$ of $\mathrm{P}, 0.60$ to $0.93 \mathrm{~g}$ of $\mathrm{Mg}, 0.60$ to $0.76 \mathrm{~g}$ of Na, and 1.51 to $1.79 \mathrm{~g}$ of $\mathrm{K}$.
\end{abstract}

Key Words: calcium, magnesium, phosphorus, potassium, requirements, sodium

\section{Introdução}

Os ovinos representam uma opção ao desenvolvimento pecuário brasileiro, por sua precocidade, prolificidade e pela adaptação às mais distintas condições ambientais e de manejo. Grande parte do interesse na exploração de ovinos deslanados se deve à adaptação desses animais às condições ambientais adversas, observando-se, nos últimos anos, crescimento acentuado, inclusive em regiões tradicionalmente criadoras de ovinos de lã. Na região Sudeste do país, onde o rebanho vem tendo grande expansão e aceitação pelos produtores, podem ser criados em áreas impróprias à agricultura e à pecuária tradicional, uma vez que esses animais são capazes de se manterem praticamente com a vegetação nativa (Lima, 1985).

A produtividade é alcançada, considerando-se os aspectos genéticos, sanitários e nutricionais, sendo

\footnotetext{
${ }_{1}^{1}$ Parte da Tese de Doutorado do primeiro autor, apresentada ao Programa de Pós-Graduação em Zootecnia da FCAV/Unesp, Campus de Jaboticabal - Projeto e Bolsa financiados pela FAPESP

2 Professor do Departamento de Zootecnia/CCA/UFPB, Campus II - 58397-000 - Areia-PB. E.mail: gonzaga@cca.ufpb.br

${ }^{3}$ Professor do Departamento de Zootecnia/FCAV/Unesp, Campus de Jaboticabal. Via de Acesso Prof. Paulo D. Castellane, Km 5 - 14884

900 - Jaboticabal-SP. E.mail: americo@fcav.unesp.br; kresende@fcav.unesp.br

${ }^{4}$ Aluno do Programa de Pós-Graduação em Zootecnia da FCAV/Unesp, Campus de Jaboticabal.

${ }^{5}$ Professor do Departamento de Medicina Veterinária/CSTR/UFCG - 58707-000 - Patos, PB.

${ }^{6}$ Aluno do Programa de Pós-Graduação em Zootecnia da FZEA/USP - Pirassununga, SP.
} 
este últimoo que reflete mais rapidamente na produção. Para o AFRC (1991), a máxima eficiência produtiva só pode ser obtida com o conhecimento adequados das exigências nutricionais dos animais e da composição química dos alimentos.

A exigência nutricional é a quantidade de um nutriente que deve ser suprida para satisfazer às necessidades de um animal saudável em um ambiente compatível com seu bem-estar (Silva, 1995). No Brasil, as exigências nutricionais de ovinos têm sido pouco estudadas e, portanto, o balanceamento das dietas é feito com base nas recomendações preconizadas pelos boletins internacionais AFRC, ARC, INRA e NRC, entre outros, desenvolvidos em países de clima temperado e que expressam as exigências de ovinos lanados. A adoção destes dados na formulação de rações para ovinos deslanados pode não proporcionar os resultados esperados, pela falta ou pelo desperdício de nutrientes, afetando a produtividade e/ou o custo de produção, pois, na determinação das exigências nutricionais, devem ser considerados raça, sexo, idade, composição corporal, alimentos disponíveis e as condições ambientais da região em que os animais são explorados. Torna-se necessário estabelecer os padrões alimentares de ovinos deslanados nas condições brasileiras, para obtenção de um sistema nutricional mais eficiente e econômico.

Portanto, objetivou-se, com este trabalho, determinar a composição corporal e estimar as exigências nutricionais de cálcio, fósforo, magnésio, sódio e potássio para mantença e ganho de peso de cordeiros Morada Nova dos 15 aos $25 \mathrm{~kg}$ de peso vivo.

\section{Material e Métodos}

Este experimento foi desenvolvido no Setor de Ovinocultura, pertencente ao Departamento de Zootecnia da Faculdade de Ciências Agrárias e Veterinárias, FCAV/Unesp, Campus de Jaboticabal, SP, localizado a $21^{\circ} 15^{\prime} 22^{\prime \prime}$ de latitude Sul e $48^{\circ} 18^{\prime} 58^{\prime \prime}$ de longitude Oeste e altitude de $595 \mathrm{~m}$.

Foram utilizados 30 cordeiros machos castrados, com peso vivo (PV) médio inicial de $15 \mathrm{~kg}$ e 70 dias de idade; seis foram abatidos no início da fase experimental, representando a composição corporal inicial, ou seja, animais-referência na metodologia do abate comparativo; seis, aos $20 \mathrm{~kg}$ (abate intermediário) e 18 foram distribuídos em três tratamentos, de acordo com a relação volumoso(V): concentrado(C) na dieta: 1) $40 \mathrm{~V}: 60 \mathrm{C}$; 2) $55 \mathrm{~V}: 45 \mathrm{C}$; e 3$) 70 \mathrm{~V}: 30 \mathrm{C}$.
Os animais distribuídos nas diferentes relações $\mathrm{V}: \mathrm{C}$ foram abatidos quando aqueles que recebiam a dieta com maior teor de concentrado atingiram $25 \mathrm{~kg}$ de PV, independentemente do peso vivo em que se encontravam os cordeiros alimentados com as demais dietas, considerando-se, portanto, o mesmo número de dias no experimento.

Os seis cordeiros do abate intermediário receberam a dieta com maior teor de concentrado e foram abatidos assim que atingiram $20 \mathrm{~kg}$ de peso vivo. Esta opção deveu-se à tentativa de melhor se estimarem as exigências de ganho de peso, realizando-se os cálculos com base nos seis animais-referência (15 kg), nos seis do abate intermediário $(20 \mathrm{~kg})$ e nos seis que receberam a dieta com maior teor de concentrado $(25 \mathrm{~kg})$, reduzindose o efeito das diferentes dietas, uma vez que as dietas 2 e 3 proporcionaram restrições qualitativas.

Foram realizadas análises laboratoriais, para determinação da composição bromatológica das dietas experimentais, conforme apresentado na Tabela 1. A dieta com menor relação $\mathrm{V}: \mathrm{C}$ foi balanceada para atender às exigências de proteína, energia metabolizável e minerais, segundo o AFRC (1993), para um ganho esperado de $150 \mathrm{~g} / \mathrm{dia}$. As demais dietas foram formuladas conforme aumento na relação volumoso:concentrado, visando promover restrição qualitativa da dieta e atender ao planejamento do ensaio de exigências nutricionais. Para a formulação das dietas experimentais, foram utilizados feno de braquiária (Brachiaria brizantha) moído e concentrado, composto por farelo de soja, milho moído, sal comum e núcleo mineral.

Os animais foram identificados e permaneceram alojados individualmente, até o abate, em gaiolas de madeira de $1,0 \mathrm{~m}^{2}$, com piso ripado suspenso, equipadas com comedouro e bebedouro, distribuídas em galpão com piso de concreto e telhas de zinco.

As dietas foram oferecidas à vontade, em duas refeições diárias, às 7 e $16 \mathrm{~h}$. O consumo total de matéria seca foi determinado pelo controle diário da quantidade de alimento fornecida e recusada.

As pesagens dos cordeiros foram realizadas a cada 14 dias, com a finalidade de monitorar o desenvolvimento ponderal dos animais. Para garantir o peso determinado de abate, os intervalos de pesagens diminuíram à medida que os animais que recebiam a dieta com maior teor de concentrado se aproximavam dos $25 \mathrm{~kg}$ de PV.

Os animais foram abatidos após jejum de alimentos sólidos e de água por 16 horas. Após pesagem, foram 
Tabela 1 - Composições percentual e bromatológica das dietas experimentais com diferentes relações volumoso(V):concentrado(C), com base na matéria seca (MS)

Table 1 - Ingredient and chemical compositions of the experimental diets with different forage $(F)$ :concentrate $(C)$ ratio on dry matter (DM) basis

\begin{tabular}{|c|c|c|c|}
\hline \multirow[t]{2}{*}{$\begin{array}{l}\text { Parâmetro } \\
\text { Item }\end{array}$} & \multicolumn{3}{|c|}{$\begin{array}{l}\text { Relação } \mathrm{V}: \mathrm{C} \\
F: C \text { ratio }\end{array}$} \\
\hline & $40: 60$ & $55: 45$ & $70: 30$ \\
\hline \multicolumn{4}{|l|}{$\begin{array}{l}\text { Composição percentual (\%MS) } \\
\text { Percent composition }(\% D M)\end{array}$} \\
\hline $\begin{array}{l}\text { Feno de braquiária (Brachiaria hay) } \\
\text { Concentrado (Concentrate) } \\
\text { Milho moído (Ground corn) } \\
\text { Farelo de soja (Soybean meal) } \\
\text { Sal iodado (Iodized salt) } \\
\text { Núcleo mineral (Mineral mix)* } \\
\end{array}$ & $\begin{array}{r}40,00 \\
60,00 \\
32,56 \\
26,74 \\
0,37 \\
0,33 \\
\end{array}$ & $\begin{array}{r}55,00 \\
45,00 \\
24,42 \\
20,06 \\
0,27 \\
0,25 \\
\end{array}$ & $\begin{array}{r}70,00 \\
30,00 \\
16,28 \\
13,37 \\
0,18 \\
0,17 \\
\end{array}$ \\
\hline \multicolumn{4}{|l|}{$\begin{array}{l}\text { Composição bromatológica (na MS) } \\
\text { Chemical composition (DM basis) }\end{array}$} \\
\hline $\begin{array}{l}\text { Matéria seca (Dry matter), }(\%) \\
\text { Proteína bruta (Crude protein), }(\%) \\
\text { Energia metabolizável }(\text { Metabolizable energy), }(\mathrm{kcal} / \mathrm{kg} \mathrm{MS}) \\
\text { Extrato etéreo (Ether extract), }(\%) \\
\text { Fibra em detergente neutro (Neutral detergent fiber }),(\%) \\
\text { Fibra em detergente ácido (Acid detergent fiber), }(\%) \\
\text { Cálcio }(\text { Calcium }),(\%) \\
\text { Fósforo }(\text { Phosphorus }),(\%) \\
\text { Magnésio }(\text { Magnesium }),(\%) \\
\text { Sódio }(\text { Sodium }),(\%) \\
\text { Potássio }(\text { Potassium }),(\%)\end{array}$ & $\begin{array}{r}88,73 \\
17,82 \\
2,34 \\
1,81 \\
45,43 \\
27,54 \\
0,52 \\
0,32 \\
0,34 \\
0,34 \\
0,91\end{array}$ & $\begin{array}{r}89,34 \\
14,11 \\
2,12 \\
1,55 \\
54,77 \\
34,33 \\
0,53 \\
0,25 \\
0,37 \\
0,27 \\
0,78\end{array}$ & $\begin{array}{r}89,95 \\
10,40 \\
1,90 \\
1,28 \\
64,11 \\
41,13 \\
0,55 \\
0,18 \\
0,40 \\
0,20 \\
0,63\end{array}$ \\
\hline
\end{tabular}

insensibilizados com descarga elétrica e mortos com secção das artérias carótidas e veias jugulares, realizando-se a coleta do sangue. $\mathrm{O}$ trato gastrintestinal foi retirado, pesado, esvaziado e pesado novamente, para determinação do peso de corpo vazio (PCV). Em seguida, todo o corpo do animal (sangue, vísceras, cabeça, extremidades dos membros, pele e carcaça) foi congelado, cortado em serra de fita, moído e homogeneizado, momento em que foram retiradas amostras de aproximadamente $500 \mathrm{~g}$. As amostras colhidas foram armazenadas em freezer para as análises laboratoriais subseqüentes.

As análises bromatológicas foram realizadas no Laboratório de Nutrição Animal - LANA e no Laboratório Central, ambos da FCAV/Unesp. As amostras dos componentes do corpo vazio foram liofilizadas por 48 horas, trituradas em liquidificador, processadas em moinho de bola e acondicionadas em recipientes de vidro hermeticamente fechados.

Nas amostras dos ingredientes da ração, das sobras, das fezes, da urina e dos componentes do corpo vazio, foram determinados os teores de matéria seca (MS), de fósforo (P), pelo método colorimétrico, e de cálcio $(\mathrm{Ca})$, magnésio $(\mathrm{Mg})$, sódio $(\mathrm{Na})$ e potássio $(\mathrm{K})$, por espectrofotometria de absorção atômica, conforme metodologia descrita por Silva \& Queiroz (2002).

A quantidade do mineral retido no corpo do animal foi determinada em função da concentração deste nutriente nas amostras analisadas. A partir desses dados, foram obtidas equações de regressão para a composição corporal.

Para se estimar o conteúdo dos minerais por quilo de corpo vazio, adotou-se a equação alométrica logaritmizada, preconizada pelo ARC (1980): $\log y=$ $a+b \log x$, em que: $\log y=\log$ aritmo do conteúdo total do mineral no corpo vazio $(\mathrm{g}) ; \mathrm{a}=$ intercepto; $\mathrm{b}=$ coeficiente de regressão do conteúdo do mineral em função do peso de corpo vazio; $\mathrm{e} \log \mathrm{x}=$ Logaritmo do peso de corpo vazio $(\mathrm{kg})$.

O conhecimento dos conteúdos corporais em diferentes pesos permitiu a estimativa da composição do 
ganho de peso dos animais nos intervalos de pesos vivos estudados (ARC, 1980). Considerou-se a diferença entre os valores de conteúdo corporal, por exemplo, de um animal de $14,5 \mathrm{~kg}$ e outro de $15,5 \mathrm{~kg}$, como a composição por $\mathrm{kg}$ de ganho de peso de um animal de $15 \mathrm{~kg}$.

As exigências líquidas de macrominerais para ganho de PCV foram obtidas derivando-se a equação alométrica logaritmizada do conteúdo corporal do mineral, em função do logaritmo do PCV, obtendo-se a equação: $\mathrm{Y}^{\prime}=\mathrm{b} \cdot 10^{\mathrm{a}} \cdot \mathrm{x}^{(\mathrm{b}-1)}$, em que: $\mathrm{Y}^{\prime}=$ exigência líquida de ganho do mineral $(\mathrm{g})$; $\mathrm{a}=$ intercepto da equação de predição do conteúdo corporal do mineral; $\mathrm{b}=$ coeficiente de regressão da equação de predição do conteúdo corporal do mineral; e $\mathrm{x}=\mathrm{PCV}(\mathrm{kg})$.

Para a conversão da exigência líquida para ganho de PCV em exigência líquida para ganho de PV, utilizou-se o fator 1,26, obtido da razão PV/PCV.

As exigências líquidas e dietéticas de macrominerais foram estimadas pelo método fatorial, preconizado pelo ARC (1980), com base nas seguintes equações:

\section{Exigências líquidas $(E L)=G+E$,}

em que: $\mathrm{G}=$ retenção diária do elemento mineral; $\mathrm{E}=$ perdas endógenas.

Exigências dietéticas $(E D)=(E L / D) \times 100$,

Tabela 2 - Fórmulas para estimativa das perdas endógenas e disponibilidade de cálcio (Ca), fósforo $(\mathrm{P})$, magnésio $(\mathrm{Mg})$, sódio $(\mathrm{Na})$ e potássio $(\mathrm{K})$

Table 2 - Estimation of endogenous losses and availability of calcium (Ca), phosphorus $(P)$, magnesium ( $\mathrm{Mg})$, sodium ( $\mathrm{Na}$ ) and potassium (K)

\begin{tabular}{llc}
\hline $\begin{array}{l}\text { Macromineral } \\
\text { Macromineral }\end{array}$ & $\begin{array}{c}\text { Perda endógena } \\
\text { Endogenous loss }\end{array}$ & $\begin{array}{c}\text { Disponibilidade (\%) } \\
\text { Availability }\end{array}$ \\
\hline $\mathrm{Ca}^{1}$ & $0,228+0,623 \times \mathrm{CMS}^{3}$ & 68 \\
$\mathrm{P}^{1}$ & $1,6 \times(-0,06+0,693 \mathrm{CMS})$ & 64 \\
$\mathrm{Mg}^{2}$ & $3,0 \mathrm{mg} / \mathrm{kg} \mathrm{PV} / \mathrm{dia}$ & 17 \\
$\mathrm{Na}^{2}$ & $25,8 \mathrm{mg} / \mathrm{kg} \mathrm{PV} / \mathrm{dia}$ & 91 \\
$\mathrm{~K}^{2}$ fecal (fecal) & $1,0 \mathrm{~g} / \mathrm{kg} \mathrm{CMS}$ & 100 \\
Urinário & $37,5 \mathrm{mg} / \mathrm{kg} \mathrm{PV} / \mathrm{dia}$ \\
Urinary & \\
Salivar & \\
Salivary & $7,0 \mathrm{mg} / \mathrm{kg} \mathrm{PV} / \mathrm{dia}$ \\
Secreções & \\
da pele & \\
Skin secretion & & \\
1 Dados obtidos do AFRC (1991) (Data obtained from AFRC (1991)). \\
2 Dados obtidos do ARC (1980) (Data obtained from ARC (1980)). \\
3 CMS = consumo de matéria seca (kg) (dry matter intake). \\
4 PV = peso vivo (kg) (body weight).
\end{tabular}

em que: $\mathrm{EL}=$ exigência líquida total; $\mathrm{D}=$ disponibilidade do elemento na dieta.

Os valores adotados de perdas endógenas e disponibilidade de macrominerais, sugeridos pelos sistemas ARC (1980) e AFRC (1991), encontram-se na Tabela 2.

As análises de variância foram realizadas pelo procedimento ANOVA do SAS (1999). Para a análise de regressão, adotou-se o seguinte modelo $\mathrm{Y}=\mathrm{a}+$ $\mathrm{bx}+\mathrm{E}$, que mostra o comportamento da variável dependente $\mathrm{Y}$ em função da variável independente $\mathrm{X}$, sendo E o erro inerente ao modelo.

\section{Resultados e Discussão}

Os resultados médios de peso vivo (PV), peso vivo ao abate (PVA), peso de corpo vazio (PCV) e composição corporal de macrominerais para cordeiros Morada Nova estão apresentados na Tabela 3.

A concentração de matéria seca no PCV acompanhou o incremento no teor de gordura com a elevação do $\mathrm{PV}$, sendo próxima aos valores encontrados por Geraseev (1998), em ovinos Santa Inês.

O aumento nas concentrações de cálcio e fósforo com o aumento do PV verificado neste trabalho difere dos resultados obtidos por Kellaway (1973), Rattray et al. (1973), Burton et al. (1974) e Thompson et al. (1988), que traalharam com animais lanados, e dos de Geraseev (1998) e Trindade (2000), que estudaram animais deslanados. Considerando-se que os animais deste experimento ainda estavam em crescimento, é fácil explicar esse aumento, pois a maioria desses elementos encontra-se nos ossos, ocorrendo redução com a maturidade, quando o osso pára de crescer (Silva, 1995).

Os elementos $\mathrm{Mg}$, Na e K mantiveram praticamente as mesmas proporções nos diferentes pesos de abate, corroborando os resultados obtidos por Trindade (2000), ao trabalhar com ovinos deslanados da raça Santa Inês.

A partir dos dados de composição corporal, foram determinadas equações de regressão do logaritmo do conteúdo corporal dos macroelementos minerais, em função do logaritmo do PCV, para cordeiros dos 15 aos $25 \mathrm{~kg}$ de PV (Tabela 4).

Os coeficientes de determinação obtidos demonstram bom ajustamento das equações $(\mathrm{P}<0,01)$, a partir das quais foram estimados os conteúdos corporais de cálcio, fósforo, magnésio, sódio e potássio, por quilograma de PCV. 
Tabela 3 - Médias e desvios-padrão do peso vivo (PV), peso ao abate (PA), peso de corpo vazio (PCV) e composição corporal de água, proteína, gordura, cálcio, fósforo, magnésio, sódio e potássio no PCV, em função da relação volumoso(V):concentrado(C) na dieta

Table 3 - Means and standard deviations of body weight, weight at slaughter, empty body weight (EBW) and body composition of water, protein, fat, calcium, phosphorus, magnesium, sodium and potassium in EBW, in response to the dietary forage $(F)$ :concentrate $(C)$ ratio

\begin{tabular}{lcccc}
\hline Parâmetro & Animais-referência & \multicolumn{3}{c}{ Relação V:C } \\
Parameter & Reference animal & \multicolumn{3}{c}{ Fatio } \\
\cline { 3 - 5 } & & $70: 30$ & $55: 45$ & $40: 60$ \\
\hline Peso vivo (kg) (Body weight) & $15,23 \pm 0,16$ & $17,60 \pm 1,50$ & $21,95 \pm 0,83$ & $25,43 \pm 0,18$ \\
Peso ao abate (kg) (Weight at slaughter) & $13,83 \pm 0,37$ & $16,12 \pm 1,56$ & $20,42 \pm 0,74$ & $23,49 \pm 0,52$ \\
Peso do corpo vazio (kg) (Empty body weight) & $11,00 \pm 0,35$ & $12,00 \pm 1,06$ & $15,95 \pm 0,92$ & $19,59 \pm 0,51$ \\
Composição corporal (Body composition), (\%) & & & & \\
Matéria seca (MS) (Dry matter - DM) & $29,86 \pm 0,84$ & $31,87 \pm 0,90$ & $33,71 \pm 0,99$ & $35,39 \pm 0,85$ \\
Gordura (Fat) & $6,72 \pm 1,66$ & $7,87 \pm 1,78$ & $10,25 \pm 1,81$ & $12,10 \pm 1,45$ \\
Cálcio (Calcium) & $1,40 \pm 0,23$ & $0,97 \pm 0,10$ & $1,42 \pm 0,13$ & $2,12 \pm 0,33$ \\
Fósforo (Phosphorus) & $0,81 \pm 0,07$ & $0,52 \pm 0,05$ & $0,80 \pm 0,04$ & $1,21 \pm 0,14$ \\
Magnésio (Magnesium) & $0,05 \pm 0,00$ & $0,05 \pm 0,00$ & $0,05 \pm 0,00$ & $0,05 \pm 0,01$ \\
Sódio (Sodium) & $0,16 \pm 0,01$ & $0,16 \pm 0,00$ & $0,15 \pm 0,01$ & $0,14 \pm 0,00$ \\
Potássio (Potassium) & $0,24 \pm 0,02$ & $0,22 \pm 0,01$ & $0,23 \pm 0,01$ & $0,22 \pm 0,01$ \\
\hline
\end{tabular}

Os resultados (Tabela 5) indicam decréscimo no conteúdo corporal em macrominerais por unidade de peso (g/kg PCV) com o aumento do PCV. Estes resultados foram próximos aos encontrados por Kellaway (1973), Geraseev (1998) e Trindade (2000).

O ARC (1980) considera a concentração de minerais no conteúdo corporal constante e independente do aumento de peso, recomendando o valor de 11,0 g de Ca e 6,0 g de P por kg PCV. Os valores médios de 13,3 g de Ca e 7,6 g de P/kg de PCV, verificados para cordeiros Morada Nova dos 15 aos 25 kg PV, são 17,3 e $21,0 \%$ inferiores aos preconizados pelo ARC (1980) e semelhantes aos reportados por Trindade (2000), para ovinos Santa Inês.

Para que o conteúdo de Ca por unidade de peso seja constante, é necessário que os tecidos muscular, ósseo e adiposo aumentem na mesma proporção, o que não aconteceu. Observou-se redução nas concentrações destes minerais com o aumento do PV, variando de 14,3 a 12,4 g de Ca por $\mathrm{kg}$ de PCV e de 8,1 a 7,2 g de $\mathrm{P}$ por $\mathrm{kg}$ de $\mathrm{PCV}$, para animais com 15 a $20 \mathrm{~kg}$ de $\mathrm{PV}$, respectivamente.

A baixa quantidade de gordura verificada nos cordeiros Morada Nova (Tabela 3), assim como em ovinos deslanados Santa Inês (Trindade, 2000; Pérez et al., 2001), implica em maiores proporções de nutrientes minerais quando comparados a ovinos lanados, uma vez que a gordura apresenta pequena quantidade de minerais, confirmando as informações de Coelho da Silva \& Leão (1979) de que no tecido adiposo praticamente não contém $\mathrm{Ca}$, enquanto nos ossos contêm de 110 a $220 \mathrm{~g}$ por $\mathrm{kg}$.

Neste trabalho, a relação Ca:P foi de 1,72, próximo aos valores preconizados pelo ARC (1980), NRC (1985) e AFRC (1991).

Houve variação nas concentrações de magnésio ( 0,47 a 0,46 g), sódio (1,6 a 1,4 g) e potássio (2,3 a 2,2 g) em cordeiros Morada Nova dos 15 aos $25 \mathrm{~kg}$ de PV. O ARC (1980) apresenta concentrações de 0,41 g de $\mathrm{Mg}, 1,1 \mathrm{~g}$ de $\mathrm{Na}$ e $1,80 \mathrm{~g}$ de $\mathrm{K}$ por $\mathrm{kg}$ de $\mathrm{PCV}$, inferiores às observadas neste trabalho.

Derivando-se as equações de regressão apresentadas na Tabela 5, foram obtidas as equações de conteúdo de $\mathrm{Ca}, \mathrm{P}, \mathrm{Mg}$, $\mathrm{Na}$ e $\mathrm{K}$ depositados por $\mathrm{kg}$ de ganho em PCV (Tabela 6).

A partir das equações apresentadas na Tabela 6 , foram estimadas as quantidades de macrominerais para ganho em PCV (Tabela 7).

A composição de cálcio e fósforo no ganho em peso, segundo o ARC (1980), é constante e independente do PV, sendo de 11,0 e $6,0 \mathrm{~g} / \mathrm{kg}$ de ganho de PCV, respectivamente. Os valores encontrados para cordeiros Morada Nova neste experimento foram semelhantes em cálcio e inferiores em fósforo, quando comparados às recomendações do ARC.

Rajaratne et al. (1990) relataram valores semelhantes de $\mathrm{Ca}(9,1 \mathrm{~g} / \mathrm{kg}$ de ganho de PCV) e inferiores de $\mathrm{P}(5,0 \mathrm{~g} / \mathrm{kg}$ de ganho de $\mathrm{PCV})$ para cordeiros 
Tabela 4 - Equações de predição para o peso de corpo vazio (PCV), em função do peso vivo (PV) e conteúdo corporal de cálcio $(\mathrm{Ca})$, fósforo $(\mathrm{P})$, magnésio $(\mathrm{Mg})$, sódio $(\mathrm{Na})$ e potássio $(\mathrm{K})$, em função do peso de corpo vazio $(\mathrm{PCV})$

Table 4 - Regression equations to predict empty body weight in relation to body weight (BW) and body content of calcium (Ca), phosphorus $(P)$, magnesium $(\mathrm{Mg})$, sodium $(\mathrm{Na})$ and potassium $(\mathrm{K})$, in relation to empty body weight (EBW)

\begin{tabular}{|c|c|c|c|}
\hline $\begin{array}{l}\text { Variável } \\
\text { Variable }\end{array}$ & $\begin{array}{c}\text { Equação de regressão } \\
\text { Regression equation }\end{array}$ & $\mathrm{R}^{2}$ & $\mathrm{CV}(\%)$ \\
\hline $\operatorname{PCV}(E B W),(\mathrm{kg})$ & $\mathrm{PCV}=-1,815431+0,88969 \mathrm{PV}^{* *}$ & 0,96 & 4,27 \\
\hline$P(g)$ & $\log \mathrm{P}=1,14430+0,77871 \log \mathrm{PCV}^{* *}$ & 0,79 & 1,86 \\
\hline $\operatorname{Mg}(g)$ & $\log \mathrm{Mg}=-0,29324+0,96353 \log \mathrm{PCV}^{* *}$ & 0,73 & 6,62 \\
\hline $\mathrm{Na}(\mathrm{g})$ & $\log \mathrm{Na}=0,45432+0,76461 \log \mathrm{PCV} * *$ & 0,94 & 1,37 \\
\hline
\end{tabular}

** Significativo a $1 \%$ de probabilidade, pelo teste $\mathrm{t}$ (Significant at $1 \%$ of probability, by $t$ test).

$\mathrm{R}^{2}$ e CV = coeficientes de determinação e variação, respectivamente $\left(R^{2}\right.$ and $C V=$ coefficients of determination and variation, respectively).

Tabela 5 - Estimativa da concentração de cálcio, fósforo, magnésio, sódio e potássio, em função do peso de corpo vazio (PCV)

Table 5 - Estimation of the concentration of calcium, phosphorus, magnesium, sodium and potassium in function of empty body weight (EBW)

\begin{tabular}{lcccccc}
\hline $\begin{array}{l}\text { Peso vivo } \\
\text { Bodyweight }\end{array}$ & PCV & $\begin{array}{c}\text { Cálcio } \\
\text { Calcium }\end{array}$ & $\begin{array}{c}\text { Fósforo } \\
\text { Phosphorus }\end{array}$ & $\begin{array}{c}\text { Magnésio } \\
\text { Magnesium }\end{array}$ & $\begin{array}{c}\text { Sódio } \\
\text { Sodium }\end{array}$ & $\begin{array}{c}\text { Potássio } \\
\text { Potassium }\end{array}$ \\
\cline { 3 - 6 } & $\mathrm{kg}$ & & & $\mathrm{g} / \mathrm{kg} \mathrm{PCV}$ \\
$\mathrm{nnnyyy}$ & & & & $0, \mathrm{~kg} E B W$ & \\
\hline 15 & 11,53 & 14,33 & 8,12 & 0,47 & 1,60 & 2,30 \\
20 & 15,98 & 13,21 & 7,55 & 0,46 & 1,48 & 2,26 \\
25 & 20,43 & 12,42 & 7,15 & & 2,23 \\
\hline
\end{tabular}

Tabela 6 - Equações de predição para o ganho de cálcio, fósforo, magnésio, sódio e potássio, em função do peso de corpo vazio (PCV)

Table 6 - Prediction equations for gain of calcium, phosphorus, magnesium, sodium and potassium, in function of empty body weight (EBW)

\begin{tabular}{lc}
$\begin{array}{l}\text { Mineral }(\mathrm{g}) \\
\text { Mineral }\end{array}$ & \multicolumn{1}{c}{$\begin{array}{c}\text { Equação } \\
\text { Equation }\end{array}$} \\
\hline $\begin{array}{l}\text { Cálcio }(\mathrm{Ca}) \\
\text { Calcium }\end{array}$ & $\mathrm{Ca}=19,79778 . \mathrm{PCV}^{-0,24987}$ \\
$\begin{array}{l}\text { Fósforo (P) } \\
\text { Phosphorus } \\
\begin{array}{l}\text { Magnésio (Mg) } \\
\text { Magnesium }\end{array}\end{array}$ & $\mathrm{P}=10,85615 . \mathrm{PCV})^{-0,22129}$ \\
$\begin{array}{l}\text { Sódio (Na) } \\
\text { Sodium } \\
\text { Potássio (K) } \\
\text { Potassium }\end{array}$ & $\mathrm{Na}=2,176048 . \mathrm{PCV}^{-0,03647}$ \\
\hline
\end{tabular}

deslanados, com 19,5 kg de PCV. Resultados semelhantes foram registrados por Trindade (2000) em cordeiros Santa Inês dos 20 aos $25 \mathrm{~kg}$ de PV. Os valores de fósforo observados variaram de 6,3 a 5,6 $\mathrm{g}$ de $\mathrm{P} / \mathrm{kg}$ de ganho de PCV e foram superiores aos citados por Geraseev (1998) e Trindade (2000).
As composições de magnésio, sódio e potássio, por $\mathrm{kg}$ de ganho de $\mathrm{PCV}$, tiveram pouca variação, apresentando valores de 0,$44 ; 1,14$ e 2,16 g, respectivamente. O ARC (1980) preconiza concentrações de 0,$4 ; 1,1$ e 1,8 g, para $\mathrm{Mg}$, $\mathrm{Na}$ e $\mathrm{K}$, respectivamente, constatando-se maiores deposições de potássio nos animais utilizados neste trabalho.

As exigências líquidas dos minerais para ganho de PV foram determinadas dividindo-se a composição de ganho de peso pelo fator da equação do PCV em função de PV (Tabela 4). A razão PV/PCV obtida neste trabalho foi 1,26 , superior à sugerida pelo ARC (1980), de 1,10.

As exigências líquidas para mantença de Ca e $\mathrm{P}$, obtidas pelas equações preconizadas pelo AFRC (1991) e que representam as perdas endógenas (Tabela 2), consideram a ingestão de matéria seca (IMS), que, por sua vez, é estimada a partir da regressão da IMS em função do PV médio, gerando a equação $\hat{Y}=-0,5569$ $+0,0684 \mathrm{PV}\left(\mathrm{R}^{2}=0,77\right)$. Estas, juntamente com as exigências líquidas para ganho de peso, são apresentadas na Tabela 8 . 
Tabela 7 - Quantidades de cálcio, fósforo, magnésio, sódio e potássio depositadas por quilograma de ganho de peso de corpo vazio (PCV), em cordeiros Morada Nova

Table 7 - Amounts of calcium, phosphorus, magnesium, sodium, and potassium storaged per $\mathrm{kg}$ of empty body weight (EBW)

\begin{tabular}{lcccccc}
\hline $\begin{array}{l}\text { Peso vivo } \\
\text { Bodyweight }\end{array}$ & PCV & $\begin{array}{c}\text { Cálcio } \\
\text { Calcium }\end{array}$ & $\begin{array}{c}\text { Fósforo } \\
\text { Phosphorus }\end{array}$ & $\begin{array}{c}\text { Magnésio } \\
\text { Magnesium }\end{array}$ & $\begin{array}{c}\text { Sódio } \\
\text { Sodium }\end{array}$ & $\begin{array}{c}\text { Potássio } \\
\text { Potassium }\end{array}$ \\
\cline { 3 - 7 } & & \multicolumn{5}{c}{$\begin{array}{c}\text { g/kg ganho de PCV } \\
\text { g/kg gained } E B W\end{array}$} \\
\hline 15 & 11,53 & 10,75 & 6,32 & 0,45 & 1,22 & 2,19 \\
20 & 15,98 & 9,91 & 5,88 & 0,44 & 1,13 & 2,15 \\
25 & 20,43 & 9,32 & 5,57 & 0,44 & 1,07 & 2,13 \\
\hline
\end{tabular}

Os valores encontrados para as exigências líquidas de ganho de Ca e P foram menores e semelhantes, respectivamente, aos observados por Geraseev (1998). Entretanto, quando se somam a estes os valores de exigência de mantença, tanto a exigência líquida total de Ca quanto a de $\mathrm{P}$ ficam maiores que as encontradas pela autora, o que pode ser explicado pela forma de obtenção das exigências de mantença, optando-se, neste trabalho, pela recomendação do AFRC (1991), que leva considera a ingestão de matéria seca (IMS).

Para o cálculo das exigências, o AFRC (1991) considera o peso à maturidade e que a deposição de $\mathrm{Ca}$ e $\mathrm{P}$ decresce à medida que o animal se torna adulto. Embora sendo a raça Morada Nova raça de maturidade precoce, os animais utilizados neste trabalho estavam em crescimento e apresentavam exigências de Ca e P ainda elevadas. Para exigência líquida de ganho, o AFRC (1991) recomenda 10,6 g de Ca/kg de PV e 6,2 $\mathrm{g}$ de $\mathrm{P} / \mathrm{kg}$ de ganho de PV, para cordeiros com $20 \mathrm{~kg}$ de $\mathrm{PV}$, valores inferiores em 15 e $16 \%$, respectivamente, aos estimados neste experimento.

As exigências líquidas de $\mathrm{Mg}, \mathrm{Na}$ e $\mathrm{K}$ apresentadas na Tabela 9 foram menores que as preconizadas pelo ARC (1980). As exigências líquidas totais de $\mathrm{Na}$ são próximas às relatadas por Geraseev (1998), para cordeiros Santa Inês de 15 a $25 \mathrm{~kg}$ de PV. O ARC (1980) sugere valores de $1,2 \mathrm{~g}$ de $\mathrm{Na} / \mathrm{kg}$ para ovinos de $45 \mathrm{~kg}$ e $1,1 \mathrm{~g}$ de $\mathrm{Na} / \mathrm{kg}$ de ganho de PCV.

As exigências líquidas de ganho de $\mathrm{K}$ foram menores que as obtidas por Geraseev (1998). Entretanto, quando se somam as exigências de mantença, esses resultados assemelham-se aos deste experimento. Essa semelhança deveu-se, provavelmente, ao menor consumo de MS, considerado no cálculo das

Tabela 8 - Exigências líquidas (EL) de cálcio e fósforo para cordeiros Morada Nova dos 15 aos 25 kg de peso vivo (PV) (g/dia)

Table 8 - Net requirements (NR) of calcium and phosphorus for Morada Nova lambs from 15 to $25 \mathrm{~kg}$ of body weight (BW) (g/day)

\begin{tabular}{|c|c|c|c|c|c|c|c|}
\hline \multirow[t]{2}{*}{$\begin{array}{l}\mathrm{PV}(\mathrm{kg}) \\
B W\end{array}$} & \multirow[t]{2}{*}{$\begin{array}{c}\mathrm{GMPD}^{1}(\mathrm{~g}) \\
A D W G\end{array}$} & \multicolumn{3}{|c|}{$\begin{array}{l}\text { Cálcio } \\
\text { Calcium }\end{array}$} & \multicolumn{3}{|c|}{$\begin{array}{c}\text { Fósforo } \\
\text { Phosphorus }\end{array}$} \\
\hline & & $\begin{array}{l}\text { Mantença }{ }^{2} \\
\text { Maintenance }\end{array}$ & $\begin{array}{l}\mathrm{EL}_{\mathrm{g}}^{3} \\
N R_{g}\end{array}$ & $\begin{array}{l}\mathrm{EL}_{\text {total }} \\
N R_{\text {total }}\end{array}$ & $\begin{array}{l}\text { Mantença }{ }^{2} \\
\text { Maintenance }\end{array}$ & $\begin{array}{l}\mathrm{EL}_{\mathrm{g}}^{3} \\
N R_{g}\end{array}$ & $\begin{array}{l}\mathrm{EL}_{\text {total }} \\
N R_{\text {total }}\end{array}$ \\
\hline \multirow{4}{*}{15} & 100 & 0,52 & 1,35 & 1,87 & 0,42 & 0,80 & 1,22 \\
\hline & 150 & 0,52 & 2,03 & 2,55 & 0,42 & 1,19 & 1,61 \\
\hline & 200 & 0,52 & 2,71 & 3,23 & 0,42 & 1,59 & 2,01 \\
\hline & 100 & 0,73 & 1,25 & 1,98 & 0,80 & 0,74 & 1,54 \\
\hline \multirow[t]{3}{*}{20} & 150 & 0,73 & 1,87 & 2,60 & 0,80 & 1,11 & 1,91 \\
\hline & 200 & 0,73 & 2,50 & 3,23 & 0,80 & 1,48 & 2,28 \\
\hline & 100 & 0,95 & 1,17 & 2,12 & 1,18 & 0,70 & 1,88 \\
\hline \multirow[t]{2}{*}{25} & 150 & 0,95 & 1,76 & 2,71 & 1,18 & 1,05 & 2,23 \\
\hline & 200 & 0,95 & 2,35 & 3,30 & 1,18 & 1,40 & 2,58 \\
\hline
\end{tabular}

\footnotetext{
1 GMPD = ganho médio de peso diário ( $A D W G$ = average daily weight gain).

2 Mantença = estimada pelo AFRC (1991) (Maintenance = estimated by AFRC, 1991).

${ }^{3} \mathrm{EL}_{\mathrm{g}}=$ Exigência líquida de ganho $\left(N R_{\mathrm{g}}=\right.$ Net requirements of gain $)$.
} 
Tabela 9 - Exigências líquidas de magnésio, sódio e potássio para cordeiros Morada Nova dos 15 aos 25 kg de peso vivo (PV) (g/dia)

Table 9 - Net requirements of magnesium, sodium, and potassium for Morada Nova lambs from 15 to $25 \mathrm{~kg}$ of live weight (LW) (g/day)

\begin{tabular}{|c|c|c|c|c|c|c|c|c|c|c|}
\hline \multirow[t]{2}{*}{$\begin{array}{l}\mathrm{PV}(\mathrm{kg}) \\
B W\end{array}$} & \multirow[t]{2}{*}{$\begin{array}{c}\mathrm{GMPD}^{1}(\mathrm{~g}) \\
A D W G\end{array}$} & \multicolumn{3}{|c|}{$\begin{array}{l}\text { Magnésio } \\
\text { Magnesium }\end{array}$} & \multicolumn{3}{|c|}{$\begin{array}{l}\text { Sódio } \\
\text { Sodium }\end{array}$} & \multicolumn{3}{|c|}{$\begin{array}{l}\text { Potássio } \\
\text { Potassium }\end{array}$} \\
\hline & & $\begin{array}{l}\text { Mantença }{ }^{2} \\
\text { Maintenance }\end{array}$ & $\begin{array}{l}\mathrm{EL}_{\mathrm{g}}^{3} \\
N R_{g}\end{array}$ & $\begin{array}{l}\mathrm{EL}_{\text {total }} \\
N R_{\text {total }}\end{array}$ & $\begin{array}{l}\text { Mantença } \\
\text { Maintenance }\end{array}$ & $\begin{array}{l}\mathrm{EL}_{\mathrm{g}}^{3} \\
N R_{g}\end{array}$ & $\begin{array}{l}\mathrm{EL}_{\text {total }} \\
N R_{\text {total }}\end{array}$ & $\begin{array}{l}\text { Mantença }{ }^{2} \\
\text { Maintenance }\end{array}$ & $\begin{array}{c}\mathrm{EL}_{\mathrm{g}}^{3} \\
N R_{g}\end{array}$ & $\begin{array}{l}\mathrm{EL}_{\text {total }} \\
N R_{\text {total }}\end{array}$ \\
\hline \multirow[t]{2}{*}{15} & 100 & 0,05 & 0,06 & 0,11 & 0,39 & 0,15 & 0,54 & 1,24 & 0,28 & 1,52 \\
\hline & 150 & 0,05 & 0,09 & 0,14 & 0,39 & 0,23 & 0,62 & 1,24 & 0,41 & 1,65 \\
\hline \multirow{2}{*}{20} & 150 & 0,06 & 0,08 & 0,14 & 0,52 & 0,21 & 0,73 & 1,80 & 0,41 & 2,21 \\
\hline & 200 & 0,06 & 0,11 & 0,17 & 0,52 & 0,29 & 0,81 & 1,80 & 0,54 & 2,34 \\
\hline \multirow[t]{3}{*}{25} & 100 & 0,08 & 0,06 & 0,14 & 0,65 & 0,14 & 0,79 & 2,37 & 0,27 & 2,64 \\
\hline & 150 & 0,08 & 0,08 & 0,16 & 0,65 & 0,20 & 0,85 & 2,37 & 0,40 & 2,77 \\
\hline & 200 & 0,08 & 0,11 & 0,19 & 0,65 & 0,27 & 0,92 & 2,37 & 0,54 & 2,91 \\
\hline
\end{tabular}

1 GMPD = ganho médio de peso diário (GMDW = average daily weight gain).

2 Mantença = estimada pelo AFRC (1991) (Maintenance = estimated by AFRC, 1991).

${ }^{3} \mathrm{EL}_{\mathrm{g}}=$ Exigência líquida de ganho $\left(N R_{\mathrm{g}}=\right.$ Net requirements of gain).

perdas endógenas, uma vez que a equação para o cálculo desta estimativa foi a mesma em ambos os trabalhos, pelo ARC (1980).

As exigências dietéticas de macrominerais obtidas neste trabalho (Tabelas 10 e 11) foram calculadas dividindo-se a exigência líquida total (Tabelas 8 e 9) pela disponibilidade de cada nutriente mineral (Tabela 2). As exigências dietéticas de $\mathrm{Ca}$ para animais com $20 \mathrm{~kg}$ de PV, ganhando $100 \mathrm{~g} / \mathrm{dia}$ (Tabela 10), foram superiores às recomendadas pelo ARC (1980) para a mesma categoria animal. O NRC (1985) sugere ingestão diária de 5,2 g de Ca para animais de $20 \mathrm{~kg}$, ganhando $200 \mathrm{~g} /$ dia, resultado $8,7 \%$ superior aos 4,75 g de Ca obtidos neste estudo.

Para fósforo, foram encontrados valores de exigências dietéticas superiores aos estimados pelo ARC (1980), possivelmente pelo uso do coeficiente de absorção de 64\%, recomendado pelo AFRC (1991), em detrimento do coeficiente de $73 \%$ preconizado pelo ARC (1980).

Ovinos Morada Nova apresentaram exigências dietéticas de $\mathrm{Ca}$ e $\mathrm{P}$ superiores às relatadas pelo $\mathrm{ARC}$ (1980) e AFRC (1991), devendo-se ter cautela na utilização destas recomendações para ovinos deslanados. As exigências dietéticas de P para animais com $20 \mathrm{~kg}$ de PV, ganhando $200 \mathrm{~g} / \mathrm{dia}$, foram $30 \%$ maiores que as recomendadas pelo NRC (1985). Annenkov (1982) e Geraseev (1998) também reportaram valores menores de exigências dietéticas para Ca e P em relação aos deste experimento.
Tabela 10 - Exigências dietéticas de cálcio e fósforo para cordeiros Morada Nova (g/dia)

Table 10 - Dietary requirements of calcium and phosphorus for Morada Nova lambs (g/day)

\begin{tabular}{lcccccc}
\hline $\begin{array}{l}\text { Peso vivo (kg) } \\
\text { Bodyweight }\end{array}$ & $\begin{array}{l}\text { Cálcio } \\
\text { Calcium }\end{array}$ \\
\hline \multicolumn{4}{c}{ GMPD $^{1}$ (g/dia) } \\
\multicolumn{4}{c}{ ADWG (g/day) } \\
\hline \multicolumn{4}{c}{$\begin{array}{c}\text { Fósforo } \\
\text { Phosphorus }\end{array}$} \\
\hline 15 & 100 & 150 & 200 & 100 & 150 & 200 \\
20 & 2,76 & 3,75 & 4,74 & 1,91 & 2,53 & 3,15 \\
25 & 3,91 & 3,83 & 4,75 & 2,41 & 2,99 & 3,57 \\
\hline 1 GMPD = ganho médio de peso diário (ADWG = average daily weight \\
gain).
\end{tabular}

As exigências dietéticas de $\mathrm{Mg}$ e $\mathrm{K}$ (Tabela 11) foram semelhantes às preconizadas pelo ARC (1980). O NRC (1985) recomenda 0,12 a $0,18 \%$ de $\mathrm{Mg}$ e 0,50 a $0,80 \%$ de $\mathrm{K}$ na matéria seca ingerida. Essas recomendações não são as mais indicadas, pois a ingestão varia entre os animais e, principalmente, com o tipo de material ingerido.

Comparando-se as exigências dietéticas totais de Mg para animais Morada Nova (Tabela 11) com as obtidas por Geraseev (1998), observam-se menores exigências neste trabalho. Para K, as exigências foram semelhantes.

As exigências dietéticas de $\mathrm{Mg}$ foram inferiores ao valor de 0,8 g/dia, recomendado por Annenkov (1982), para cordeiros com $20 \mathrm{~kg}$ de PV e ganho diário de $100 \mathrm{~g}$. 


\begin{tabular}{|c|c|c|c|c|c|c|c|c|c|}
\hline \multirow[t]{3}{*}{$\begin{array}{l}\text { Peso vivo }(\mathrm{kg}) \\
\text { Body weight }\end{array}$} & \multicolumn{3}{|c|}{$\begin{array}{l}\text { Magnésio } \\
\text { Magnesium }\end{array}$} & \multicolumn{3}{|c|}{$\begin{array}{l}\text { Sódio } \\
\text { Sodium }\end{array}$} & \multicolumn{3}{|c|}{$\begin{array}{l}\text { Potássio } \\
\text { Potassium }\end{array}$} \\
\hline & \multicolumn{9}{|c|}{$\begin{array}{l}\mathrm{GMPD}^{1} \text { (g/dia) } \\
A D W G \text { (g/day) }\end{array}$} \\
\hline & 100 & 150 & 200 & 100 & 150 & 200 & 100 & 150 & 200 \\
\hline 15 & 0,60 & 0,77 & 0,93 & 0,60 & 0,68 & 0,76 & 1,51 & 1,65 & 1,79 \\
\hline 20 & 0,68 & 0,85 & 1,01 & 0,72 & 0,80 & 0,88 & 2,07 & 2,21 & 2,34 \\
\hline 25 & 0,77 & 0,93 & 1,09 & 0,86 & 0,93 & 1,01 & 2,63 & 2,77 & 2,90 \\
\hline
\end{tabular}

A ingestão diária de Na sugerida pelo ARC (1980) é de 0,69 e 0,97 g por dia para animais com 15 e $25 \mathrm{~kg}$ de $\mathrm{PV}$, respectivamente. A exigência dietética de $\mathrm{Na}$ deste trabalho foi $5 \%$ menor que a preconizada pelo ARC (1980). O NRC (1985), por outro lado, recomenda ingestão diária de 0,09 a $0,18 \%$ de Na com base na matéria seca. As exigências dietéticas de $\mathrm{Na}$ foram 0,8 g para animais com $20 \mathrm{~kg}$ de $\mathrm{PV}$, valor $2,4 \%$ inferior ao observado por Geraseev (1998) e 6,3\% superior aos níveis obtidos por Trindade (2000), ambos com a raça deslanada Santa Inês.

É importante ressaltar que a disponibilidade dos minerais modifica de acordo com os alimentos utilizados na dieta. Segundo Resende (1989), esta disponibilidade é afetada pelas inter-relações entre minerais, categoria animal e outros. Portanto, deve-se utilizar as exigências líquidas, transformando-as em exigências dietéticas de acordo com a dieta a ser utilizada. O autor afirma que é importante atentar-se para a forma de se expressar a exigência dietética, pois muitos o fazem como porcentagem da matéria seca ingerida, e enfatiza a grande variabilidade na ingestão de matéria seca, indicando como melhor maneira de expressar essas exigências a quantidade do mineral ingerido por dia.

\section{Conclusões}

A composição corporal variou de 14,33 a 12,42 g de $\mathrm{Ca} ; 8,12$ a 7,15 g de P; 0,47 a 0,46 g de $\mathrm{Mg} ; 1,60$ a 1,40 g de $\mathrm{Na}$ e de 2,30 a 2,23 g de K por kg de peso de corpo vazio.

As exigências líquidas de ganho de cordeiros Morada Nova variaram de 13,54 a 11,74 mg de Ca; 7,96 a 7,02 mg de P; 0,57 a 0,55 mg de Mg; 1,54 a 1,35 $\mathrm{mg}$ de $\mathrm{Na}$ e de 2,75 a 2,68 mg de K por g de PV ganho.
Recomenda-se ingestão diária de 2,76 a 3,12 g de $\mathrm{Ca} ; 1,91$ a 2,95 g de P; 0,60 a 0,77 g de Mg; 0,60 a 0,86 $\mathrm{g}$ de $\mathrm{Na}$ e de 1,51 a 2,63 g de K para cordeiros dos 15 aos $25 \mathrm{~kg}$ de $\mathrm{PV}$, ganhando $100 \mathrm{~g} / \mathrm{dia}$.

As exigências líquidas de Ca e P para ganho de peso foram 15 e $20 \%$ superiores, respectivamente, aos valores recomendados pelo AFRC (1991). Para $\mathrm{Mg}$, $\mathrm{Na}$ e K, foram encontrados valores ligeiramente inferiores aos recomendados pelo ARC (1980).

As exigências dietéticas de Ca e $\mathrm{P}$ para ovinos Morada Nova nas condições desta pesquisa foram superiores às recomendações do ARC (1980), NRC (1985) e AFRC (19991).

\section{Literatura Citada}

AGRICULTURAL AND FOOD RESEARCH COUNCIL AFRC. Technical committee on responses to nutrients. A reappraisal of the calcium and phosphorus requirements of sheep and cattle. Report 6. Nutrition Abstracts and Reviews. Série B, v.61, n.9, p.573-612, 1991.

AGRICULTURAL AND FOOD RESEARCH COUNCIL AFRC. Necesidades energéticas y proteicas de los rumiantes. Zaragoza: Acribia, 1993. 175p.

AGRICULTURAL RESEARCH COUNCIL - ARC. The nutrient requirements of ruminant livestock. London, Farnham Royal: Commonwealth Agricultural Bureaux, 1980. 351p.

ANNENKOV, B.N. Mineral feeding of sheep. In: GEORGIEVSKII, V.I.; ANNENKOV, B.N.; SAMOKHIN, V.I. (Eds.) Mineral nutrition of animals. London: Butterworths, 1982. p.321-54.

BURTON, J.H.; ANDERSON, M.; REID, J.T. Some biological aspects of partial starvation. The effect of weight loss and regrowth on body composition in sheep. British Journal Nutrition, v.32, n.3, p.515-27, 1974.

GERASEEV, L.C. Composição corporal e exigências em macrominerais ( $\mathrm{Ca}, \mathrm{P}, \mathrm{Mg}, \mathrm{K}$ e $\mathrm{Na}$ ) de cordeiros Santa Inês. Lavras: Universidade Federal de Lavras, 1998. 99p. Tese (Doutorado em Zootecnia) - Universidade Federal de Lavras, 1998. 
KELLAWAY, R.C. The effects of plane of nutrition, genotype and sex on growth, body composition and wool production in grazing sheep. Journal of Agricultural Science, v.80, n.1, p.17-27, 1973.

LIMA, F.A.M. Desempenho de ovinos deslanados no Nordeste brasileiro e planos de melhoramento para o futuro. In: SIMPÓSIO INTERNACIONAL PRODUÇÃO ANIMAL, 1., 1985, Ribeirão Preto. Anais... Ribeirão Preto: Sociedade Brasileira de Melhoramento Animal, 1995. p.55-67.

NATIONAL RESEARCH COUNCIL - NRC. Nutrient requirements of sheep. 6.ed. Washington: National Academy Press, 1985. 99p.

PÉREZ, J.R.O.; GERASEEV, L.C.; SANTOS, C.L. et al. Composição corporal e exigências nutricionais de cálcio e fósforo de cordeiros Santa Inês em crescimento. Pesquisa Agropecuária Brasileira, v.36, n.5, p.815-822, 2001.

RAJARATNE, A.A.J.; SCOTT, D.; THOMPSON, J.K. et al. The effect of variation in dietary calcium supply on the phosphorus requirements of growing lambs. Animal Production, v.51, n.1, p.135-42, 1990.

RATTRAY, P.V.; GARRET, W.N.; MEYER, H.H. et al. Body and carcass composition of targhee and finn-targhee lambs. Journal Animal Science, v.37, n.4, p.892-7, 1973.

RESENDE, K.T. Métodos de estimativa da composição corporal e exigências nutricionais de proteína, energia, e macroelementos inorgânicos de caprinos em crescimento. Viçosa, MG: Universidade Federal de Viçosa, 1989. 130p. Tese (Doutorado em Zootecnia) - Universidade Federal de Viçosa, 1989.
STATISTICAL ANALYSES SYSTEM - SAS. User's guide. Versão 8.0. North Carolina: 1999.

SILVA, D.J.; QUEIROZ, A.C. Análise de alimentos - métodos químicos e biológicos. 3.ed. Viçosa, MG: Universidade Federal de Viçosa, 2002. 235p.

SILVA, J.F.C. Exigência de macroelementos inorgânicos para bovinos: O sistema ARC/AFRC e a experiência no Brasil. In: SIMPÓSIO INTERNACIONAL SOBRE EXIGÊNCIAS NUTRICIONAIS DE RUMINANTES, 1995, Viçosa, MG. Anais... Viçosa, MG: Universidade Federal de Viçosa, 1995. p.467-504.

SILVA, J.F.C.; LEÃO, M.I. Fundamentos de nutrição de ruminantes. Piracicaba: Livroceres, 1979. 380p.

THOMPSON, D.J.; WERNER, J.C. Cálcio, fósforo e flúor na nutrição animal. In: SIMPÓSIO LATINO AMERICANO SOBRE PESQUISA EM NUTRIÇÃO MINERAL DE RUMINANTES EM PASTAGENS, 1976, Belo Horizonte. Anais... Belo Horizonte: UFMG, UFV, ESAL, EPAMIG, 1976. p.1-10.

TRINDADE, I.A.C.M. Composição corporal e exigências nutricionais em macrominerais de ovinos lanados e deslanados, em crescimento. Jaboticabal: Universidade Estadual Paulista, 2000. 66p. Dissertação (Mestrado em Zootecnia) - Universidade Estadual Paulista, 2000.

Recebido em: 30/11/03 Aceito em: 08/07/05 